\title{
Effectiveness of a Third Dose of Pfizer-BioNTech and Moderna Vaccines in Preventing COVID-19 Hospitalization Among Immunocompetent and Immunocompromised Adults — United States, August-December 2021
}

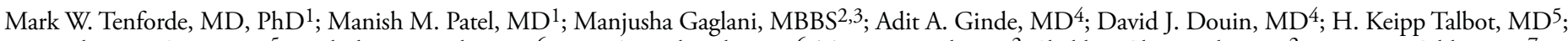
Jonathan D. Casey, MD5; Nicholas M. Mohr, MD ${ }^{6}$; Anne Zepeski, PharmD ${ }^{6}$; Tresa McNeal, MD²; Shekhar Ghamande, MD²; Kevin W. Gibbs, MD ${ }^{7}$; D. Clark Files, $\mathrm{MD}^{7}$; David N. Hager, MD, PhD ${ }^{8}$; Arber Shehu, MD ${ }^{8}$; Matthew E. Prekker, MD ; Heidi L. Erickson, MD ${ }^{9}$; Michelle N. Gong, MD ${ }^{10}$; Amira Mohamed, MD ${ }^{10}$; Nicholas J. Johnson, MD 11 ; Vasisht Srinivasan, MD ${ }^{11}$; Jay S. Steingrub, MD ${ }^{12}$; Ithan D. Peltan, MD ${ }^{13}$; Samuel M. Brown, MD 13 ; Emily T. Martin, $\mathrm{PhD}^{14}$; Arnold S. Monto, MD ${ }^{14}$; Akram Khan, $\mathrm{MD}^{15}$; Catherine L. Hough, $\mathrm{MD}^{15}$; Laurence W. Busse, MD ${ }^{16}$; Abhijit Duggal, MD ${ }^{17}$; Jennifer G. Wilson, MD ${ }^{18}$; Nida Qadir, MD ${ }^{19}$; Steven Y. Chang, MD, PhD ${ }^{19}$; Christopher Mallow, MD ${ }^{20}$; Carolina Rivas ${ }^{20}$; Hilary M. Babcock, MD 21 ; Jennie H. Kwon, DO ${ }^{21}$; Matthew C. Exline, MD²2; Mena Botros, MD² ; Adam S. Lauring, MD, PhD ${ }^{23}$; Nathan I. Shapiro, MD 24 ; Natasha Halasa, MD5;

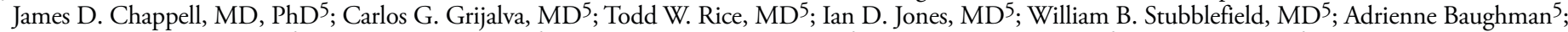
Kelsey N. Womack, $\mathrm{PhD}^{5}$; Jillian P. Rhoads, PhD ${ }^{5}$; Christopher J. Lindsell, PhD 5 ; Kimberly W. Hart, MA ${ }^{5}$; Yuwei Zhu, MD ${ }^{5}$; Eric A. Naioti, MPH ${ }^{1}$; Katherine Adams, $\mathrm{MPH}^{1}$; Nathaniel M. Lewis, $\mathrm{PhD}^{1}$; Diya Surie, $\mathrm{MD}^{1}$; Meredith L. McMorrow, MD ${ }^{1}$; Wesley H. Self, MD ${ }^{5}$; IVY Network
\end{abstract}

COVID-19 mRNA vaccines (BNT162b2 [Pfizer-BioNTech] and mRNA-1273 [Moderna]) provide protection against infection with SARS-CoV-2, the virus that causes COVID-19, and are highly effective against COVID-19-associated hospitalization among eligible persons who receive 2 doses $(1,2)$. However, vaccine effectiveness (VE) among persons with immunocompromising conditions* is lower than that among immunocompetent persons (2), and VE declines after several months among all persons (3). On August 12, 2021, the Food and Drug Administration (FDA) issued an emergency use authorization (EUA) for a third mRNA vaccine dose as part of a primary series $\geq 28$ days after dose 2 for persons aged $\geq 12$ years with immunocompromising conditions, and, on November 19, 2021 , as a booster dose for all adults aged $\geq 18$ years at least 6 months after dose 2 , changed to $\geq 5$ months after dose 2 on January 3, 2022 (4,5,6). Among 2,952 adults (including 1,385 COVID-19 case-patients and 1,567 COVID-19-negative controls) hospitalized at 21 U.S. hospitals during August 19December 15, 2021, effectiveness of mRNA vaccines against COVID-19-associated hospitalization was compared between adults eligible for but who had not received a third vaccine dose $(1,251)$ and vaccine-eligible adults who received a third dose $\geq 7$ days before illness onset (312). Among 1,875 adults without immunocompromising conditions (including 1,065 [57\%] unvaccinated, 679 [36\%] 2-dose recipients, and 131 [7\%] 3-dose [booster] recipients), VE against COVID-19

\footnotetext{
* Persons with immunocompromising conditions defined by the IVY Network included those with one or more of the following: active solid organ cancer (active cancer was defined as treatment for the cancer or newly diagnosed cancer in the past 6 months); active hematologic cancer (such as leukemia, lymphoma, or myeloma); HIV infection without AIDS; AIDS; congenital immunodeficiency syndrome; previous splenectomy; prior solid organ, stem cell, or bone marrow transplant; use of immunosuppressive medication; systemic lupus erythematosus; rheumatoid arthritis; psoriasis; scleroderma; or inflammatory bowel disease, including Crohn's disease or ulcerative colitis.
}

hospitalization was higher among those who received a booster dose (97\%; 95\% CI $=95 \%-99 \%)$ compared with that among 2-dose recipients (82\%; 95\% CI $=77 \%-86 \%)(\mathrm{p}<0.001)$. Among 1,077 adults with immunocompromising conditions (including 324 [30\%] unvaccinated, 572 [53\%] 2-dose recipients, and 181 [17\%] 3-dose recipients), VE was higher among those who received a third dose to complete a primary series (88\%; 95\% CI = 81\%-93\%) compared with 2-dose recipients (69\%; 95\% CI $=57 \%-78 \%)(\mathrm{p}<0.001)$. Administration of a third COVID-19 mRNA vaccine dose as part of a primary series among immunocompromised adults, or as a booster dose among immunocompetent adults, provides improved protection against COVID-19-associated hospitalization.

During August 19-December 15, 2021, a period in which the SARS-CoV-2 B.1.617.2 (Delta) variant was predominant, adults admitted to 21 hospitals in 18 states within the Influenza or Other Viruses in the Acutely Ill Network (IVY Network) who received testing for SARS-CoV-2 were included in a VE analysis. The analysis start date of August 19, 2021, was one week after the EUA of a third dose for persons with immunocompromising conditions. VE against COVID-19 hospitalization was compared between two groups: 1) adults who had completed a 2-dose mRNA vaccination series and were eligible for but had not received a third dose $^{\dagger}$; and 2) adults who were eligible for and had received a third dose $\geq 7$ days before illness onset. VE was calculated for 2-dose and 3 -dose recipients by comparing odds of antecedent vaccination between COVID-19 case-patients and control patients who

\footnotetext{
$\dagger$ During the surveillance period, third doses were recommended $\geq 28$ days after dose 2 if the patient had an immunocompromising condition to complete a primary series or at least 6 months after dose 2 as a booster dose if the patient did not have an immunocompromising condition. The booster dose for Moderna $(0.25 \mathrm{~mL})$ is one half the dose used for the primary series $(0.5 \mathrm{~mL})$. The third dose to complete a primary series in persons with immunocompromising conditions is the same as the first 2 doses $(0.5 \mathrm{~mL})$.
} 
did not have COVID-19. Case-patients had COVID-19-like illness ${ }^{\S}$ and received positive $S A R S-C o V-2$ test results by a nucleic acid amplification test (NAAT) or antigen test within 10 days of illness onset. Control patients were hospitalized with or without COVID-19-like illness and received negative SARS-CoV-2 test results by NAAT.

Patients or their proxies were interviewed regarding patient demographic and clinical characteristics, and medical record searches were completed to collect information about chronic medical conditions. Information about receipt of prior COVID-19 vaccination doses, including dates, locations, and vaccine product received, was obtained through self-report and review of source documentation (including state vaccination registries, medical records, and vaccination cards). A patient was considered to be vaccinated if vaccination could be verified through source documentation or by self-report, including dates and location. Three vaccination groups were considered: 1) unvaccinated patients, who had received no COVID-19 vaccine doses before illness onset; 2) 2-dose mRNA vaccine recipients, who were eligible for but had not received a third vaccine dose or had received a third dose $<7$ days before illness onset; and 3) 3-dose mRNA vaccine recipients, who received a third dose $\geq 7$ days before illness onset. Patients were excluded if they were admitted to the hospital $\leq 7$ days after EUA authorization of a third dose, received one or more vaccine doses but did not qualify for inclusion in the 2-dose or 3-dose vaccine group, 9 received a non-mRNA vaccine (e.g., Ad26.COV2.S [Janssen (Johnson \& Johnson)]), or if verification of vaccination was pending.

VE against COVID-19-associated hospitalization was estimated using logistic regression, comparing the odds of being vaccinated versus being unvaccinated among case-patients and controls using the equation $\mathrm{VE}=100 \times(1$ - adjusted odds ratio). The regression model included case-patient or control status as the outcome variable and an indicator vari $\neg$ able for vaccination group (unvaccinated, 2-dose recipient, or 3-dose recipient), and was adjusted for admission date, region of

\footnotetext{
$\$$ COVID-19-like illness was defined as having one or more of the following: fever, cough, shortness of breath, loss of taste, loss of smell, use of respiratory support for the acute illness, or new pulmonary findings on chest imaging consistent with pneumonia.

Satients were excluded if they received a third COVID-19 mRNA vaccine dose before the FDA EUA (before August 12, 2021 for patients with immunocompromising conditions or before September 22, 2021 for patients without immunocompromising conditions [when a single booster dose of the Pfizer-BioNTech vaccine was authorized]), were admitted to the hospital before $\leq 7$ days had elapsed since EUA authorization (before August 19, 2021 for patients with immunocompromising conditions or before September 29, 2021 for patients without immunocompromising conditions) or received a third dose before recommended during the surveillance period ( $<28$ days after dose 2 for patients with immunocompromising conditions or $<180$ days after dose 2 for patients without immunocompromising conditions).
}

hospital (U.S. Department of Health and Human Services or U.S. Census Bureau), age group (18-49, 50-64, or $\geq 65$ years), sex, and self-reported race and ethnicity. Separate models were generated for immunocompetent adults and adults with immunocompromising conditions. To compare VE among 2-dose versus 3-dose mRNA vaccine recipients, post hoc comparisons were performed with the pwcompare function in Stata with a two-sided significance threshold of $p<0.05$. Analyses were conducted using Stata software (version 16.0; StataCorp). This activity was determined to be public health surveillance by each participating site and CDC and was conducted consistent with applicable federal law and CDC policy.**

During August 19-December 15, 2021, the IVY Network enrolled 4,094 adults aged $\geq 18$ years. After excluding 1,142 patients ( 619 because they did not belong to an included vaccination group, 386 because the patients had received a nonmRNA vaccine or vaccination verification was incomplete, and 137 because they met other exclusion criteria), 2,952 hospitalized patients were included (1,385 case-patients and 1,567 non-COVID-19 controls). Among all participants, median age was 62 years, $49 \%$ of patients were female, $58 \%$ were non-Hispanic White, and $36 \%$ had an immunocompromising condition. Among the 1,385 case-patients, 931 (67\%), $408(29 \%)$, and $46(3 \%)$ had received 0,2 , and 3 mRNA vaccine doses, respectively. Among 1,567 non-COVID-19 controls, 458 (29\%), 843 (54\%), and 266 (17\%) had received 0,2 , and 3 mRNA vaccine doses, respectively. Among patients without immunocompromising conditions (Table 1), 2- and 3 -dose recipients were similar in terms of age (median $=69$ and 72 years, respectively) but differed in self-reported race/ ethnicity distribution (a higher percentage of non-Hispanic White persons were among 3-dose recipients; $p=0.008$ ) and U.S. Census Bureau region of the admitting hospital $(\mathrm{p}=0.030)$. Two-dose recipients were less likely to report working in health care settings (5\%) than were 3-dose recipients $(10 \%)(p=0.023)$ and were more likely to be enrolled as a COVID-19 case-patient (31\%) than were 3-dose recipients $(8 \%)(\mathrm{p}<0.001)$. Among patients with immunocompromising conditions (Table 2), 2-dose recipients were more likely to be enrolled as a case-patient (34\%) than were 3-dose recipients $(20 \%)(\mathrm{p}<0.001)$. VE against COVID-19 hospitalization among adults without immunocompromising conditions was $82 \%(95 \% \mathrm{CI}=77 \%-86 \%)$ for 2 doses and $97 \%$ $(95 \% \mathrm{CI}=95 \%-99 \%)$ for 3 doses $(\mathrm{p}<0.001)$ (Table 3). VE against COVID-19 hospitalization among adults with immunocompromising conditions was $69 \%(95 \% \mathrm{CI}=57 \%-78 \%)$ for 2 doses and $88 \%(95 \%$ CI $=81 \%-93 \%)$ for 3 doses

\footnotetext{
** 45 C.F.R. part 46.102(1)(2), 21 C.F.R. part 56; 42 U.S.C. Sect. 241(d); 5 U.S.C. Sect. 552a; 44 U.S.C. Sect. 3501 et seq.
} 
TABLE 1. Characteristics of vaccine effectiveness analysis participants without immunocompromising conditions, including case-patients hospitalized with COVID-19 and controls hospitalized without COVID-19, by mRNA vaccination group - 21 hospitals, ${ }^{*} 18$ U.S. states, August-December 2021

\begin{tabular}{|c|c|c|c|c|}
\hline \multirow[b]{2}{*}{ Characteristic } & \multicolumn{3}{|c|}{ Vaccination group, no./Total no. (\%) ${ }^{\dagger}$} & \multirow{2}{*}{$\begin{array}{l}\text { P-value for } \\
\text { comparison of } \\
\text { 2-dose versus } \\
\text { 3-dose group? }\end{array}$} \\
\hline & $\begin{array}{l}\text { Unvaccinated } \\
(n=1,065)\end{array}$ & $\begin{array}{l}2 \text { mRNA vaccine doses } \\
\quad(n=679)^{\dagger}\end{array}$ & $\begin{array}{l}3 \text { mRNA vaccine doses } \S \\
(n=131)\end{array}$ & \\
\hline COVID-19 case-patients & $744 / 1,065(70)$ & $212 / 679(31)$ & $10 / 131(8)$ & $<0.001$ \\
\hline $\begin{array}{l}\text { Age group, yrs } \\
18-49 \\
50-64 \\
\geq 65\end{array}$ & $\begin{array}{l}454 / 1,065(43) \\
327 / 1,065(31) \\
284 / 1,065(27)\end{array}$ & $\begin{array}{r}64 / 679(9) \\
179 / 679(26) \\
436 / 679(64)\end{array}$ & $\begin{array}{r}9 / 131(7) \\
29 / 131(22) \\
93 / 131(71)\end{array}$ & 0.31 \\
\hline Female sex & $486 / 1,065(46)$ & $329 / 679(48)$ & $67 / 131(51)$ & 0.57 \\
\hline $\begin{array}{l}\text { Race/Ethnicity** } \\
\text { White, non-Hispanic } \\
\text { Black, non-Hispanic } \\
\text { Any race, Hispanic } \\
\text { Non-Hispanic, all other races } \\
\text { Unknown }\end{array}$ & $\begin{array}{r}566 / 1,065(53) \\
220 / 1,065(21) \\
195 / 1,065(18) \\
57 / 1,065(5) \\
27 / 1,065(3)\end{array}$ & $\begin{array}{r}409 / 679(60) \\
134 / 679(20) \\
85 / 679(13) \\
37 / 679(5) \\
14 / 679(2)\end{array}$ & $\begin{array}{r}100 / 131(76) \\
16 / 131(12) \\
10 / 131(8) \\
2 / 131(2) \\
3 / 131(2)\end{array}$ & 0.008 \\
\hline $\begin{array}{l}\text { U.S. Census region } \\
\text { Northeast } \\
\text { Midwest } \\
\text { South } \\
\text { West }\end{array}$ & $\begin{array}{l}176 / 1,065(17) \\
215 / 1,065(20) \\
391 / 1,065(37) \\
283 / 1,065(27)\end{array}$ & $\begin{array}{l}160 / 679(24) \\
151 / 679(22) \\
210 / 679(31) \\
158 / 679(23)\end{array}$ & $\begin{array}{l}20 / 131(15) \\
32 / 131(24) \\
35 / 131(27) \\
44 / 131(34)\end{array}$ & 0.030 \\
\hline LTCF resident ${ }^{\dagger \dagger}$ & $25 / 1,004(2)$ & $71 / 663(11)$ & $17 / 120(14)$ & 0.27 \\
\hline $\begin{array}{l}\text { Employed } \\
\text { Health care worker }\end{array}$ & $\begin{array}{r}343 / 813(42) \\
21 / 813(3)\end{array}$ & $\begin{array}{r}104 / 556(19) \\
26 / 556(5)\end{array}$ & $\begin{array}{l}23 / 96(24) \\
10 / 96(10)\end{array}$ & $\begin{array}{l}0.23 \\
0.023\end{array}$ \\
\hline Reported $\geq 1$ previous hospitalization in the last year & $267 / 947(28)$ & $298 / 598(50)$ & $50 / 119(42)$ & 0.12 \\
\hline No. of chronic medical condition types (IQR) & $1(0-2)$ & $2(1-3)$ & $2(1-3)$ & 0.10 \\
\hline $\begin{array}{l}\text { Self-reported previous laboratory-confirmed } \\
\text { SARS-CoV-2 infection }\end{array}$ & $68 / 1,063(6)$ & $36 / 679(5)$ & $7 / 131(5)$ & 0.98 \\
\hline $\begin{array}{l}\text { Vaccine product received } \\
\text { Pfizer-BioNTech } \\
\text { Moderna } \\
\text { Both products }\end{array}$ & $\begin{array}{l}\text { NA } \\
\text { NA } \\
\text { NA }\end{array}$ & $\begin{array}{r}386 / 679(57) \\
288 / 679(42) \\
5 / 679(0.7)\end{array}$ & $\begin{array}{r}93 / 131(71) \\
35 / 131(27) \\
3 / 131(2)\end{array}$ & 0.001 \\
\hline $\begin{array}{l}\text { Interval between second vaccine dose and illness } \\
\text { onset, days, median, (IQR) }\end{array}$ & NA & $225(203-248)$ & $257(240-276)$ & $<0.001$ \\
\hline $\begin{array}{l}\text { Interval between second and third vaccine dose, days, } \\
\text { median (IQR) }\end{array}$ & NA & NA & $230(211-248)$ & NA \\
\hline $\begin{array}{l}\text { Interval between third vaccine dose and illness onset, } \\
\text { days, median (IQR) }\end{array}$ & NA & NA & $25(13-36)$ & NA \\
\hline
\end{tabular}

Abbreviations: LTCF = long-term care facility; NA = not applicable.

* Hospitals by U.S. Census region included Northeast: Baystate Medical Center (Springfield, Massachusetts), Beth Israel Deaconess Medical Center (Boston, Massachusetts), Montefiore Medical Center (New York City borough of the Bronx, New York); Midwest: University of lowa Hospitals \& Clinics (lowa City, lowa), University of Michigan Hospital (Ann Arbor, Michigan), Hennepin County Medical Center (Minneapolis, Minnesota), Barnes-Jewish Hospital (St. Louis, Missouri), Cleveland Clinic (Cleveland, Ohio), The Ohio State University Wexner Medical Center (Columbus, Ohio); South: Vanderbilt University Medical Center (Nashville, Tennessee), University of Miami Medical Center (Miami, Florida), Emory University Hospital (Atlanta, Georgia), The Johns Hopkins Hospital (Baltimore, Maryland), Atrium Health Wake Forest Baptist Medical Center (Winston-Salem, North Carolina), Baylor Scott \& White Medical Center (Temple, Texas); West: Stanford University Medical Center (Palo Alto, California), Ronald Reagan UCLA Medical Center (Los Angeles, California), UCHealth University of Colorado Hospital (Aurora, Colorado), Oregon Health \& Science University Hospital (Portland, Oregon), Intermountain Medical Center (Murray, Utah), University of Washington Medical Center (Seattle, Washington).

† Three vaccination groups were included; unvaccinated patients received no COVID-19 vaccine doses before the date of current illness onset; 2-dose recipients received 2 doses of an mRNA COVID-19 vaccine with the second dose received $\geq 180$ days before the date of illness onset; 3 -dose recipients received a third (booster) dose of vaccine with $\geq 180$ days between the second and third dose and $\geq 7$ days between the date of dose 3 receipt and illness onset. Among those who received a third dose with mRNA-1273 from Moderna, one half a dose $(0.25 \mathrm{~mL})$ was recommended for the third dose.

$\S$ Dose 3 received $\geq 6$ months after dose 2 .

" Comparisons were made between 2-dose (primary series only) and 3-dose recipients (booster) groups using Pearson's chi-square testing for categorical variables and the non-parametric Wilcoxon rank-sum test for continuous variables.

** Race and ethnic groups self-reported.

t+ LTCFs included residence in a nursing home, assisted living home, or rehab hospital or other subacute or chronic facility before the hospital admission. 
TABLE 2. Characteristics of vaccine effectiveness analysis participants with immunocompromising conditions, ${ }^{*}$ including case-patients hospitalized with COVID-19 and controls hospitalized without COVID-19, by mRNA vaccination group - 21 hospitals, ${ }^{\dagger} 18$ U.S. states, AugustDecember 2021

\begin{tabular}{|c|c|c|c|c|}
\hline \multirow[b]{2}{*}{ Characteristic } & \multicolumn{3}{|c|}{ Vaccination group, no./Total no. $(\%)^{\S}$} & \multirow{2}{*}{$\begin{array}{c}\text { P-value for } \\
\text { comparison of } 2 \text {-dose } \\
\text { versus 3-dose group* }\end{array}$} \\
\hline & $\begin{array}{l}\text { Unvaccinated } \\
\quad(n=324)\end{array}$ & $\begin{array}{l}\text { mRNA vaccine, } \\
2 \text { doses }(n=572)^{\dagger}\end{array}$ & $\begin{array}{l}\text { mRNA vaccine, } 3 \text { doses"; } \\
\qquad(n=181)\end{array}$ & \\
\hline COVID-19 case patients & $187 / 324(58)$ & $196 / 572(34)$ & $36 / 181(20)$ & $<0.001$ \\
\hline $\begin{array}{l}\text { Age group, yrs } \\
18-49 \\
50-64 \\
\geq 65\end{array}$ & $\begin{array}{r}100 / 324(31) \\
131 / 324(40) \\
93 / 324(29)\end{array}$ & $\begin{array}{r}84 / 572(15) \\
197 / 572(34) \\
291 / 572(51)\end{array}$ & $\begin{array}{r}20 / 181(11) \\
55 / 181(30) \\
106 / 181(59)\end{array}$ & 0.17 \\
\hline Female sex & $168 / 324(52)$ & $303 / 572(53)$ & $81 / 181(45)$ & 0.054 \\
\hline $\begin{array}{l}\text { Race/Ethnicity }{ }^{\dagger \dagger} \\
\text { White, non-Hispanic } \\
\text { Black, non-Hispanic } \\
\text { Any race, Hispanic } \\
\text { Non-Hispanic, all other races } \\
\text { Unknown }\end{array}$ & $\begin{array}{r}176 / 324(54) \\
82 / 324(25) \\
55 / 324(17) \\
8 / 324(2) \\
3 / 324(0.9)\end{array}$ & $\begin{array}{r}329 / 572(58) \\
113 / 572(20) \\
97 / 572(17) \\
25 / 572(4) \\
8 / 572(1)\end{array}$ & $\begin{array}{r}133 / 181(73) \\
24 / 181(13) \\
14 / 181(8) \\
9 / 181(5) \\
1 / 181(0.6)\end{array}$ & 0.001 \\
\hline $\begin{array}{l}\text { U.S. Census region } \\
\text { Northeast } \\
\text { Midwest } \\
\text { South } \\
\text { West }\end{array}$ & $\begin{array}{r}48 / 324(15) \\
72 / 324(22) \\
145 / 324(45) \\
59 / 324(18)\end{array}$ & $\begin{array}{r}94 / 572(16) \\
131 / 572(23) \\
206 / 572(36) \\
141 / 572(25)\end{array}$ & $\begin{array}{l}24 / 181(13) \\
48 / 181(27) \\
61 / 181(34) \\
48 / 181(27)\end{array}$ & 0.56 \\
\hline LTCF resident $^{\S \S}$ & $8 / 316(3)$ & $18 / 558(3)$ & $3 / 178(2)$ & 0.28 \\
\hline $\begin{array}{l}\text { Employed } \\
\text { Health care worker }\end{array}$ & $\begin{array}{r}62 / 258(24) \\
9 / 258(3)\end{array}$ & $\begin{array}{r}108 / 497(22) \\
13 / 497(3)\end{array}$ & $\begin{array}{r}34 / 159(21) \\
5 / 159(3)\end{array}$ & $\begin{array}{l}0.93 \\
0.72\end{array}$ \\
\hline $\begin{array}{l}\text { Reported } \geq 1 \text { previous hospitalization in the } \\
\text { last year }\end{array}$ & $153 / 307(50)$ & $317 / 540(59)$ & $96 / 164(59)$ & 0.97 \\
\hline No. of chronic medical condition types (IQR) & $3(2-4)$ & $3(2-4)$ & $3(3-4)$ & 0.88 \\
\hline $\begin{array}{l}\text { Self-reported previous laboratory-confirmed } \\
\text { SARS-CoV-2 infection }\end{array}$ & $33 / 324(10)$ & $38 / 572(7)$ & $12 / 181(7)$ & 1.00 \\
\hline $\begin{array}{l}\text { Vaccine product received } \\
\text { Pfizer-BioNTech } \\
\text { Moderna } \\
\text { Both products }\end{array}$ & $\begin{array}{l}\text { NA } \\
\text { NA } \\
\text { NA }\end{array}$ & $\begin{array}{r}334 / 572(58) \\
238 / 572(42) \\
0 / 572(-)\end{array}$ & $\begin{array}{r}120 / 181(66) \\
57 / 181(31) \\
4 / 181(2)\end{array}$ & $<0.001$ \\
\hline $\begin{array}{l}\text { Interval between second vaccine dose and illness } \\
\text { onset, days, median (IQR) }\end{array}$ & NA & $178(142.5-213)$ & $226(199-251)$ & $<0.001$ \\
\hline $\begin{array}{l}\text { Interval between second and third vaccine dose, } \\
\text { days, median (IQR) }\end{array}$ & NA & NA & $178(148-202)$ & NA \\
\hline $\begin{array}{l}\text { Interval between third vaccine dose and illness } \\
\text { onset, days, median (IQR) }\end{array}$ & NA & NA & $40(21-68)$ & NA \\
\hline
\end{tabular}

Abbreviations: LTCF = long-term care facility; NA = not applicable.

* Immunocompromising conditions included having one or more of the following: active solid organ cancer (active cancer defined as treatment for the cancer or newly diagnosed cancer in the past 6 months); active hematologic cancer (such as leukemia, lymphoma, or myeloma); HIV infection without AIDS; AIDS; congenital immunodeficiency syndrome; prior splenectomy; prior solid organ, stem cell, or bone marrow transplant; immunosuppressive medication; systemic lupus erythematosus; rheumatoid arthritis; psoriasis; scleroderma; or inflammatory bowel disease, including Crohn's disease or ulcerative colitis. Among 2-dose vaccine recipients with immunocompromising conditions (572), $274(48 \%)$ had active cancer, $156(27 \%)$ were on immunosuppressive medications, 118 ( $21 \%)$ had a history of solid organ transplant, and $146(26 \%)$ had another immunocompromising condition; among 3-dose vaccine recipients with immunocompromising conditions (181), 88 (49\%) had active cancer, 74 (41\%) were on immunosuppressive medications, 65 (36\%) had a history of solid organ transplant, and 20 (11\%) had another immunocompromising condition.

${ }^{\dagger}$ Hospitals by U.S. Census region included Northeast: Baystate Medical Center (Springfield, Massachusetts), Beth Israel Deaconess Medical Center (Boston, Massachusetts), Montefiore Medical Center (New York City borough of the Bronx, New York); Midwest: University of lowa Hospitals \& Clinics (lowa City, lowa), University of Michigan Hospital (Ann Arbor, Michigan), Hennepin County Medical Center (Minneapolis, Minnesota), Barnes-Jewish Hospital (St. Louis, Missouri), Cleveland Clinic (Cleveland, Ohio), The Ohio State University Wexner Medical Center (Columbus, Ohio); South: Vanderbilt University Medical Center (Nashville, Tennessee), University of Miami Medical Center (Miami, Florida), Emory University Hospital (Atlanta, Georgia), The Johns Hopkins Hospital (Baltimore, Maryland), Atrium Health Wake Forest Baptist Medical Center (Winston-Salem, North Carolina), Baylor Scott \& White Medical Center (Temple, Texas); West: Stanford University Medical Center (Palo Alto, California), Ronald Reagan UCLA Medical Center (Los Angeles, California), UCHealth University of Colorado Hospital (Aurora, Colorado), Oregon Health \& Science University Hospital (Portland, Oregon), Intermountain Medical Center (Murray, Utah), University of Washington Medical Center (Seattle, Washington).

$\S$ Three vaccination groups were included; unvaccinated patients received no COVID-19 vaccine doses before the date of current illness onset; 2 -dose recipients received 2 doses of an mRNA COVID-19 vaccine with the second dose received $\geq 28$ days before the date of illness onset; 3 -dose recipients received 3 doses of vaccine to complete a primary vaccine series with $\geq 28$ days between the second and third dose and $\geq 7$ days between the date of dose 3 receipt and illness onset.

" Dose 3 received $\geq 28$ days after dose 2 .

** Comparisons were made between 2-dose and 3-dose recipient groups using Pearson's chi-square testing for categorical variables and the nonparametric Wilcoxon rank-sum test for continuous variables.

t+ Race and ethnic groups self-reported.

$\S \S$ LTCFs included residence in a nursing home, assisted living home, or rehab hospital or other subacute or chronic facility before the hospital admission. 
$(\mathrm{p}<0.001)$. In a sensitivity analysis among patients with moderately to severely immunocompromising conditions defined by $\mathrm{CDC}^{\dagger \dagger}(7)$, VE was $65 \%$ (95\% CI $\left.=49 \%-76 \%\right)$ for receipt of 2 doses, and $87 \%(95 \% \mathrm{CI}=78 \%-92 \%)$ for receipt of 3 doses $(\mathrm{p}<0.001)$.

\section{Discussion}

In a multistate network, adults vaccinated with 2 or 3 doses of a COVID-19 mRNA vaccine were protected against COVID-19-associated hospitalization. Significantly higher VE was observed in adults who received a third mRNA vaccine dose either as part of a primary vaccine series (immunocompromised persons) or as a booster dose (immunocompetent persons) compared with those who had received 2 doses. These findings underscore the importance of immunocompromised adults obtaining a third $m R N A$ vaccine dose $\geq 28$ days after the second vaccine dose and of immunocompetent adults receiving a third (booster) dose currently recommended $\geq 5$ months after the second dose. $\$ \$$

\footnotetext{
$\dagger \dagger$ The definition adapted from CDC definitions of moderately to severely immunocompromising conditions included having one or more of the following: active solid organ cancer (active cancer defined as treatment for the cancer or newly diagnosed cancer in the past 6 months), active hematologic cancer (such as leukemia, lymphoma, or myeloma), AIDS, congenital immunodeficiency syndrome, previous solid organ, stem cell, or bone marrow transplant, or active immunosuppressive medication use.

$\$ \$$ https://www.cdc.gov/vaccines/covid-19/clinical-considerations/covid-19vaccines-us.html
}

This study was conducted during a period of SARS-CoV-2 Delta variant predominance (8). VE against the B.1.1.529 (Omicron) variant of SARS-CoV-2 might be lower than for other variants that have circulated widely, possibly because of immune evasion. Early evidence suggests that a third mRNA vaccine dose elicits markedly stronger neutralizing antibody responses to the Omicron variant compared with responses to 2 vaccine doses (9), and increases VE against severe disease following infection with the Omicron variant (10). The effectiveness of 3 doses of COVID-19 mRNA vaccines against a range of disease severity associated with the Omicron variant needs to be carefully evaluated in different populations.

The findings in this report are subject to at least six limitations. First, 2-dose and 3-dose vaccine recipients were similar in terms of most demographic and clinical characteristics but might have differed with respect to exposure risk for SARS-CoV-2 infection or risk factors for severe COVID-19. Therefore, residual or unmeasured confounding was possible. Second, VE associated with newly emergent variants, including Omicron, was not assessed. Third, VE was not assessed against SARS-CoV-2 infection or mild illness. Fourth, most 3-dose $m R N A$ vaccine recipients were vaccinated within several weeks of enrollment and durability of protection will require future analysis. Fifth, VE associated with a fourth mRNA vaccine dose, recommended as a booster dose in immunocompromised individuals $\geq 5$ months after dose 3 , was not assessed. Finally, although medical centers in 18 states were included in the

TABLE 3. Effectiveness of 2-dose and 3-dose regimens of COVID-19 mRNA vaccines against COVID-19 hospitalization among adults with and without immunocompromising conditions - 21 hospitals, 18 U.S. states, ${ }^{*}+{ }^{\dagger}$ August-December 2021

\begin{tabular}{|c|c|c|c|c|c|}
\hline \multirow[b]{2}{*}{ Subgroup } & \multicolumn{2}{|c|}{$\begin{array}{l}\text { Vaccinated versus unvaccinated, } \\
2 \text { doses }\end{array}$} & \multicolumn{2}{|c|}{$\begin{array}{c}\text { Vaccinated versus unvaccinated, } \\
3 \text { doses }\end{array}$} & \multirow{2}{*}{$\begin{array}{l}\text { P-value for VE comparison } \\
\text { for 2-dose versus } \\
\text { 3-dose recipients } \\
\S\end{array}$} \\
\hline & $\begin{array}{l}\text { No. vaccinated/ } \\
\text { Total no. (\%) }\end{array}$ & $\operatorname{VE}(95 \% \mathrm{Cl}) *$ & $\begin{array}{l}\text { No. vaccinated/ } \\
\text { Total no. (\%) }\end{array}$ & VE $(95 \% \mathrm{Cl})^{*}$ & \\
\hline \multicolumn{6}{|c|}{ Patients without immunocompromising conditions } \\
\hline COVID-19 case-patients & 212/956 (22) & $82(77-86)$ & 10/754 (1) & $97(95-99)$ & $<0.001$ \\
\hline Control patients & $467 / 788(59)$ & & $121 / 442(27)$ & & \\
\hline \multicolumn{6}{|c|}{ Patients with immunocompromising conditions } \\
\hline COVID-19 case-patients & $196 / 383(51)$ & $69(57-78)$ & $36 / 223(16)$ & $88(81-93)$ & $<0.001$ \\
\hline Control patients & $376 / 513(73)$ & & $145 / 282(51)$ & & \\
\hline
\end{tabular}

Abbreviation: $\mathrm{VE}=$ vaccine effectiveness.

* VE was estimated using logistic regression, comparing the odds of being vaccinated with the Moderna or Pfizer-BioNTech COVID-19 vaccine product versus being unvaccinated among case-patients and controls using the equation VE $=100 \times(1$-adjusted odds ratio). The regression model included three categories for vaccination status: unvaccinated, vaccinated with 2 doses of an mRNA vaccine, or vaccinated with 3 doses of an mRNA vaccine. VE was calculated separately comparing 2-dose recipients to unvaccinated controls and 3-dose recipients to unvaccinated controls. Models were adjusted for date of hospital admission (biweekly intervals), U.S. Department of Health and Human Services region of hospital, age group (18-49, 50-64, or $\geq 65$ years), sex, and race/ethnicity (non-Hispanic White, non-Hispanic Black, Hispanic of any race, non-Hispanic Other, or unknown).

${ }^{\dagger}$ Hospitals by U.S. Department of Health and Human Services region included: Region 1: Baystate Medical Center (Springfield, Massachusetts), Beth Israel Deaconess Medical Center (Boston, Massachusetts); Region 2: Montefiore Medical Center (New York City borough of the Bronx, New York); Region 3: The Johns Hopkins Hospital (Baltimore, Maryland); Region 4: Vanderbilt University Medical Center (Nashville, Tennessee), University of Miami Medical Center (Miami, Florida), Emory University Hospital (Atlanta, Georgia), Atrium Health Wake Forest Baptist Medical Center (Winston-Salem, North Carolina); Region 5: University of Michigan Hospital (Ann Arbor, Michigan), Hennepin County Medical Center (Minneapolis, Minnesota), Cleveland Clinic (Cleveland, Ohio), The Ohio State University Wexner Medical Center (Columbus, Ohio); Region 6: Baylor Scott \& White Medical Center (Temple, Texas); Region 7: University of lowa Hospitals \& Clinics (lowa City, lowa), Barnes-Jewish Hospital (St. Louis, Missouri); Region 8: UCHealth University of Colorado Hospital (Aurora, Colorado), Intermountain Medical Center (Murray, Utah); Region 9: Stanford University Medical Center (Palo Alto, California), Ronald Reagan UCLA Medical Center (Los Angeles, California); Region 10: Oregon Health \& Sciences University Hospital (Portland, Oregon), University of Washington Medical Center (Seattle, Washington).

$\S$ Post-hoc pairwise comparisons of VE for 2-dose versus 3-dose vaccine recipients was evaluated using the pwcompare function in Stata software (version 16; StataCorp); a two sided p-value $<0.05$ indicated a significant difference in VE between groups. 


\section{Summary}

What is already known about this topic?

For adults aged $\geq 18$ years who received 2 doses of an mRNA COVID-19 vaccine, third doses are recommended. However, the associated benefits in preventing COVID-19 hospitalization are incompletely understood.

What is added by this report?

In a study of hospitalized adults, compared with receipt of 2 mRNA COVID-19 vaccine doses, receipt of a third dose increased vaccine effectiveness against hospitalization among adults without and with immunocompromising conditions, from $82 \%$ to $97 \%$ and from $69 \%$ to $88 \%$, respectively.

What are the implications for public health practice?

Administration of a third COVID-19 mRNA vaccine dose as part of a primary series among immunocompromised adults, or as a booster dose among immunocompetent adults, provides improved protection against COVID-19-associated hospitalization.

analysis, patients might not be representative of the general U.S. population.

Among adults with and without immunocompromising conditions who were eligible to receive a third dose of COVID-19 mRNA vaccine, third doses were found to increase protection beyond that of a 2-dose vaccination series for the prevention of COVID-19 hospitalization. Administration of a third COVID-19 mRNA vaccine dose as part of a primary series among immunocompromised adults, or as a booster dose among immunocompetent adults, provides improved protection against COVID-19 hospitalization.

\section{IVY Network}

Nicole Calhoun, Baylor Scott \& White Health; Kempapura Murthy, Baylor Scott \& White Health; Judy Herrick, Baylor Scott \& White Health; Amanda McKillop, Baylor Scott \& White Health; Eric Hoffman, Baylor Scott \& White Health; Martha Zayed, Baylor Scott \& White Health; Michael Smith, Baylor Scott \& White Health; Ryan Kindle, Baystate Medical Center; Lori-Ann Kozikowski, Baystate Medical Center; Lesley De Souza, Baystate Medical Center; Scott Ouellette, Baystate Medical Center; Sherell Thornton-Thompson, Baystate Medical Center; Omar Mehkri, Cleveland Clinic; Kiran Ashok, Cleveland Clinic; Susan Gole, Cleveland Clinic; Alexander King, Cleveland Clinic; Bryan Poynter, Cleveland Clinic; Caitlin ten Lohuis, Emory University; Nicholas Stanley, Emory University; Audrey Hendrickson, Hennepin County Medical Center; Sean Caspers, Hennepin County Medical Center; Walker Tordsen, Hennepin County Medical Center; Olivia Kaus, Hennepin County Medical Center; Tyler Scharber, Hennepin County Medical Center; Jeffrey Jorgensen, Intermountain Medical Center; Robert Bowers, Intermountain Medical Center; Jennifer King, Intermountain Medical Center; Valerie Aston, Intermountain Medical Center; Richard E. Rothman, Johns Hopkins University; Harith Ali, Johns
Hopkins University; Rahul Nair, Montefiore Medical Center; Jen-Ting (Tina) Chen, Montefiore Medical Center; Sarah Karow, Ohio State University; Emily Robart, Ohio State University; Paulo Nunes Maldonado, Ohio State University; Maryiam Khan, Ohio State University; Preston So, Ohio State University; Olivia Krol, Oregon Health \& Science University; Jesus Martinez Oregon Health \& Science University; Zachary Zouyed Oregon Health \& Science University; Michael Acosta Oregon Health \& Science University; Reihaneh Bazyarboroujeni, Oregon Health \& Science University; Haeun Jung, Oregon Health \& Science University; Raju Reddy, Oregon \& Health Science University; Richard Zhang, Oregon Health \& Science University; Alexandra Jun Gordon, Stanford University; Joe Levitt, Stanford University; Cynthia Perez, Stanford University; Anita Visweswaran, Stanford University; Jonasel Roque, Stanford University; Sukantha Chandrasekaran, University of California, Los Angeles; Trevor Frankel, University of California, Los Angeles; Omai Garner, University of California, Los Angeles; Jennifer Goff, UCHealth University of Colorado Hospital; David Huynh, UCHealth University of Colorado Hospital; Kelly Jensen, UCHealth University of Colorado Hospital; Conner Driver, UCHealth University of Colorado Hospital; Michael Carricato, UCHealth University of Colorado Hospital; Ian Chambers, UCHealth University of Colorado Hospital; Paul Nassar, University of Iowa; Lori Stout, University of Iowa; Zita Sibenaller, University of Iowa; Alicia Walter, University of Iowa; Jasmine Mares, University of Iowa; Spenser Pfannenstiel, University of Iowa; Hayley Gershengorn, University of Miami; EJ McSpadden, University of Michigan; Rachel Truscon, University of Michigan; Lara Thomas, University of Michigan; Ramsay Bielak, University of Michigan; Weronika Damek Valvano, University of Michigan; Rebecca Fong, University of Michigan; William J. Fitzsimmons, University of Michigan; Christopher Blair, University of Michigan; Julie Gilbert, University of Michigan; Christine D. Crider, University of Washington; Kyle A. Steinbock, University of Washington; Thomas C. Paulson, University of Washington; Layla A. Anderson, University of Washington; Christy Kampe, Vanderbilt University Medical Center; Jakea Johnson, Vanderbilt University Medical Center; Laura L. Short, Vanderbilt University Medical Center; Lauren J. Ezzell, Vanderbilt University Medical Center; Margaret E. Whitsett, Vanderbilt University Medical Center; Rendie E. McHenry, Vanderbilt University Medical Center; Samarian J. Hargrave, Vanderbilt University Medical Center; Marica Blair, Vanderbilt University Medical Center; Jennifer L. Luther, Vanderbilt University Medical Center; Claudia Guevara Pulido, Vanderbilt University Medical Center; Bryan P. M. Peterson, Vanderbilt University Medical Center; Mary LaRose, Wake Forest University; Leigha Landreth, Wake Forest University; Madeline Hicks, Wake Forest University; Lisa Parks, Wake Forest University; Jahnavi Bongu, Washington University; David McDonald, Washington University; Candice Cass, Washington University; Sondra Seiler, Washington University; David Park, Washington University; Tiffany Hink, Washington University; Meghan Wallace, Washington University; Carey-Ann Burnham, Washington University; Olivia G. Arter, Washington University. 
Corresponding author: Mark W. Tenforde, media@cdc.gov.

${ }^{1}$ CDC COVID-19 Emergency Response Team; ${ }^{2}$ Baylor Scott \& White Health, Temple, Texas; ${ }^{3}$ Texas A\&M University College of Medicine, Temple, Texas; ${ }^{4}$ University of Colorado School of Medicine, Aurora, Colorado; ${ }^{5}$ Vanderbilt University Medical Center, Nashville, Tennessee; ${ }^{6}$ University of Iowa, Iowa City, Iowa; ${ }^{7}$ Wake Forest University Baptist Medical Center, Winston-Salem, North Carolina; ${ }^{8}$ The Johns Hopkins Hospital, Baltimore, Maryland; ${ }^{9}$ Hennepin County Medical Center, Minneapolis, Minnesota; ${ }^{10}$ Montefiore Medical Center, Albert Einstein College of Medicine, New York City borough of the Bronx, New York; ${ }^{11}$ University of Washington School of Medicine, Seattle, Washington; ${ }^{12}$ Baystate Medical Center, Springfield, Massachusetts; ${ }^{13}$ Intermountain Medical Center and University of Utah, Salt Lake City, Utah; ${ }^{14}$ University of Michigan School of Public Health, Ann Arbor, Michigan; ${ }^{15}$ Oregon Health \& Science University Hospital, Portland, Oregon; ${ }^{16}$ Emory University School of Medicine, Atlanta, Georgia; ${ }^{17}$ Cleveland Clinic, Cleveland, Ohio; ${ }^{18}$ Stanford University School of Medicine, Palo Alto, California; ${ }^{19}$ Ronald Reagan-UCLA Medical Center, Los Angeles, California; ${ }^{20}$ University of Miami, Miami, Florida; ${ }^{21}$ Washington University, St. Louis, Missouri; ${ }^{22}$ Ohio State University Wexner Medical Center, Columbus, Ohio; ${ }^{23}$ University of Michigan School of Medicine, Ann Arbor, Michigan; ${ }^{24}$ Beth Israel Deaconess Medical Center, Boston, Massachusetts.

All authors have completed and submitted the International Committee of Medical Journal Editors form for disclosure of potential conflicts of interest. Samuel M. Brown reports personal fees from Hamilton, institutional fees from Faron Pharmaceuticals and Sedana, grants from Janssen, the National Institutes of Health (NIH), the Department of Defense (DoD), book royalties from Oxford University and Brigham Young University, outside the submitted work. Steven Y. Chang was a speaker for La Jolla Pharmaceuticals in 2018 and consulted for PureTech Health in 2020. James D. Chappell reports grants from NIH during the conduct of the study. Abhijit Duggal reports grants from NIH and participation on a steering committee for ALung Technologies, Inc., outside the submitted work. Matthew C. Exline reports support from Abbott Labs for sponsored talks, outside the submitted work. D. Clark Files reports personal consultant fees from Cytovale and is a data and safety monitoring board (DSMB) member from Medpace, outside the submitted work. Manjusha Gaglani reports grants from CDC-Vanderbilt University Medical Center for the submitted work, CDC-Abt Associates, CDC-Westat, Janssen and Pfizer, outside the submitted work. Adit A. Ginde reports grants from NIH, DoD, AbbVie, and Faron Pharmaceuticals, outside the submitted work. Michelle N. Gong reports grants from NIH and the Agency for Healthcare Research and Quality (AHRQ), DSMB membership fees from Regeneron, outside the submitted work. Carlos G. Grijalva reports consultancy fees from Pfizer, Merck, and Sanofi-Pasteur; grants from Campbell Alliance/ Syneos Health, NIH, the Food and Drug Administration, AHRQ, and Sanofi, outside the submitted work. David N. Hager reports salary support from Incyte Corporation and EMPACT Precision Medicine via Vanderbilt University Medical Center and grants from NHLBI, outside the submitted work. Natasha Halasa reports grants and nonfinancial support from Sanofi, and grants from Quidel outside the submitted work. Akram Khan reports grants from United Therapeutics, Johnson \& Johnson, Ely Lilly, and GlaxoSmithKline, outside the submitted work. Adam S. Lauring reports personal fees from Sanofi and Roche, outside the submitted work. Christopher J. Lindsell reports grants from NIH, DoD, and the Marcus Foundation; contract fees from bioMerieux, Endpoint LLC, and Entegrion Inc, outside the submitted work; in addition, Dr. Lindsell has a patent for risk stratification in sepsis and septic shock issued. Emily T. Martin reports personal fees from Pfizer and grants from Merck, outside the submitted work. Ithan D. Peltan reports grants from NIH, Janssen Pharmaceuticals and institutional support from Asahi Kasei Pharma and Regeneron, outside the submitted work. Todd W. Rice reports personal fees from Cumberland Pharmaceuticals, Inc, Cytovale, Inc., and Sanofi, Inc., outside the submitted work. Wesley H. Self reports consulting fees from Aeprio Pharmaceuticals and Merck, outside the submitted work. No other potential conflicts of interest were disclosed.

\section{References}

1. Thompson MG, Stenehjem E, Grannis S, et al. Effectiveness of COVID-19 vaccines in ambulatory and inpatient care settings. N Engl J Med 2021;385:1355-71. Epub September 8, 2021. PMID:34496194 https://doi.org/10.1056/NEJMoa2110362

2 Tenforde MW, Self WH, Adams K, et al.; Influenza and Other Viruses in the Acutely Ill (IVY) Network. Association between mRNA vaccination and COVID-19 hospitalization and disease severity. JAMA 2021;326:2043-54. PMID:34734975 https://doi.org/10.1001/ jama.2021.19499

3. Tartof SY, Slezak JM, Fischer H, et al. Effectiveness of mRNA BNT162b2 COVID-19 vaccine up to 6 months in a large integrated health system in the USA: a retrospective cohort study. Lancet 2021;398:1407-16. PMID:34619098 https://doi.org/10.1016/S0140-6736(21)02183-8

4. Food and Drug Administration. Coronavirus (COVID-19) update: FDA takes multiple actions to expand use of Pfizer-BioNtech COVID-19 vaccine. Silver Spring, MD: US Department of Health and Human Services, Food and Drug Administration; 2022. https://www.fda.gov/ news-events/press-announcements/coronavirus-covid-19-update-fdatakes-multiple-actions-expand-use-pfizer-biontech-covid-19-vaccine

5. Food and Drug Administration. Coronavirus (COVID-19) update: FDA authorizes additional vaccine dose for certain immunocompromised individuals. Silver Spring, MD: US Department of Health and Human Services, Food and Drug Administration; 2021. https://www.fda.gov/ news-events/press-announcements/coronavirus-covid-19-update-fdaauthorizes-additional-vaccine-dose-certain-immunocompromised.

6. Food and Drug Administration. Coronavirus (COVID-19) update: FDA expands eligibility for COVID-19 vaccine boosters. Silver Spring, MD: US Department of Health and Human Services, Food and Drug Administration; 2021. https://www.fda.gov/news-events/pressannouncements/coronavirus-covid-19-update-fda-expands-eligibilitycovid-19-vaccine-boosters.

7. CDC. COVID-19: COVID-19 vaccines for moderately or severely immunocompromised people. Atlanta, GA: US Department of Health and Human Services, CDC; 2021. Accessed January 6, 2021.https:// www.cdc.gov/coronavirus/2019-ncov/vaccines/recommendations/ immuno.html

8. CDC. CDC COVID data tracker. Atlanta, GA: US Department of Health and Human Services, CDC; 2021. Accessed December 14, 2021. https://covid.cdc.gov/covid-data-tracker/\#cases_casesper100klast7days

9. Nemet I, Kliker L, Lustig Y, et al. Third BNT162b2 vaccination neutralization of SARS-CoV-2 Omicron infection. N Engl J Med 2021;NEJMc2119358. PMID:34965337 https://doi.org/10.1056/ NEJMc2119358

10. Thompson MG, Natarajan K, Irving SA, et al. Effectiveness of a third dose of mRNA vaccines against COVID-19-associated emergency department and urgent care encounters and hospitalizations among adults during periods of delta and omicron variant predominanceVISION Network, 10 states, August 2021-January 2022. MMWR Morb Mortal Wkly Rep Epub January 21, 2022. http://dx.doi.org/10.15585/ mmwr.mm7104e3 